\title{
MODELLING OF TOOL LIFE, TORQUE AND THRUST FORCE IN DRILLING: A NEURO-FUZZY APPROACH
}

\author{
Roy, S. S. \\ Department of Mechanical Engineering, \\ National Institute of Technology, Durgapur, West Bengal-713209, India \\ E-Mail: ssroy@nitdgp.ac.in
}

\begin{abstract}
This paper presents the application of neuro-fuzzy approach for modelling tool life, torque and thrust force in drilling operation for set of given process parameters, namely cutting speed, feed rate and drill diameter. The proposed approach uses a hybrid-learning algorithm i.e., combination of the back-propagation gradient descent method and least squares method, to identify premise and consequent parameters of the first-order Sugeno-fuzzy inference system. The least square method is used to optimize the consequent parameters with the premise parameters fixed. Once the optimal consequent parameters are found, the back-propagation algorithm gradient descent method is used to adjust optimally the premise parameters corresponding to the fuzzy set in the input domain. The predicted tool life, torque and thrust force values obtained from neuro-fuzzy system were compared with the experimental data. This comparison indicates that the proposed approach can produce optimal knowledge base of fuzzy system for predicting tool life, torque and thrust force in drilling operation.

(Received in August 2009, accepted in November 2009. This paper was with the author 1 month for 1 revision.)
\end{abstract}

Key Words: Tool Life, Torque, Thrust Force, Neuro-Fuzzy Approach, Drilling

\section{INTRODUCTION}

Drilling is a most commonly used machining operation in the manufacturing industry. Therefore, modelling of tool life, torque and thrust force in milling process plays an important role in the manufacturing industry. Several attempts were made, in past, to model tool wear and drill force by using experimental investigation approach and design of experiment method. Choudhury [1] used a regression model to measure the flank and corner wear of a drill bit. A regression model was developed by Xiaoli [2] for monitoring the tool wear based on current signal of spindle motor and feed motor. Ertunc [3] used the hidden Markov model for monitoring drill wear using cutting force signal. Liu [4] used the polynomial network for in-process prediction of corner wear in drilling. Force signal were considered to monitor on-line drill wear [5]. In recent years, the use of adaptive learning tools to construct the machining database for associating the cutting parameters with cutting performance has gradually been accepted as a reliable, effective modelling technique. This is because adaptive learning tools have an excellent ability to learn and to interpolate the complicated relationships between cutting parameters and cutting performance. A biologically inspired network called abductive network was adopted by Lee [6] to model the drilling operation. A back-propagation neural network (BPNN) has been used by various researchers to construct a prediction model of tool wear in drilling operation. In this connection work of Lin and Ting [7], Panda et al. [8], Singh et al. [9], Panda et al. [10] are significant. Tsao [11] utilized the radial basis function network (RBFN) and adaptive based radial basis function network (ARBFN) to predict flank wear. Recently, Tsao and Hocheng [12] used Taguchi method and radial basis function network (RBFN) for prediction and 
evaluation of thrust force and surface roughness in drilling of composite material. Panda et al. [13] introduced the fuzzy rule into the BPNN model for predicting the flank wear of drill bit. Sanjay et al. [14] estimated drill wear by statistical method and artificial neural network. Panda et al. [15] used both RBFN and BPNN for prediction of flank wear of high speed steel (HSS) drill in a cast iron work piece using cutting parameters and sensor signals. In this work, the input-output relationships in drilling are modelled using neuro-fuzzy approach in which the optimum knowledge base i.e. combination of data base and rule base of the fuzzy system are designed using neural networks, so that the tool life, torque and thrust force in drilling can be modelled for set of input process parameters namely, cutting speed, feed rate and drill diameter.

The paper is structured in the following manner. The theory of neuro-fuzzy modelling is introduced first. The modelling methodology using neuro-fuzzy system is then described. The simulation results and comparison experimental results in modelling of tool life, torque and thrust force are presented thereafter. Finally, the paper concludes with a summary of the study.

\section{DESCRIPTION OF NEURO-FUZZY MODELLING}

\subsection{Fuzzy modelling}

Fuzzy set theory was developed by Prof. L.A. Zadeh [16] to capture the imprecise modes of reasoning employed in an environment characterized by uncertainty and vagueness. The system of concepts, principles and methods dealing with approximate reasoning by the use of fuzzy sets concepts is fuzzy logic. It can be seen as an extension of set-theoretic bivalent logic. The fuzzy set operations defined on fuzzy sets such as intersection, union and complement, can also be employed in fuzzy logic to represent respectively the 'and', 'or' and 'not' connections. A fuzzy inference system employing fuzzy 'if-then' rules can model the qualitative aspects of human knowledge and reasoning processes without employing precise quantitative analysis. This fuzzy modelling was first explored systematically by Takagi and Sugeno [17]. A fuzzy inference system is composed of a rule base, containing a number of fuzzy If-then rules; a database, which defines the membership functions of fuzzy sets used in fuzzy rules; a decision-making unit which performs inference operations on the rules; a defuzzification interface, which transforms fuzzy results into a crisp output. The rule base and database are jointly referred to as the knowledge base. The steps of fuzzy reasoning performed by fuzzy inference system are: i) Compare the input variables with the membership functions on the premise part to obtain the membership values of each linguistic label. ii) Combine (through a specific T-norm operator) the membership values on the premise part to get firing strength (weight) of each rule. iii) Generate the qualified consequent of each rule depending on the firing strength. iv) Aggregate the qualified consequents to produce a crisp output. This study incorporates a Takagi-Sugeno fuzzy model, in which the output of each If-then rule is a linear combination of input variables plus a constant term. The final output is input weighted average of each rule's output and is obtained by union of the output fuzzy sets.

\subsection{Adaptive neuro-fuzzy modelling}

The Adaptive Neuro-Fuzzy system is a Takagi-Sugeno fuzzy model put in the framework of adaptive systems to facilitate learning and adaptation [18]. Such framework makes the adaptive neuro-fuzzy modelling more systematic and less reliant on expert knowledge. To illustrate the architecture of Adaptive Neuro-Fuzzy system, for simplicity, we assume that the 
fuzzy inference system under consideration has two inputs ( $v$ and $d$ ) and one output $(f)$. The architecture presented in this section can easily be extended to three input and one output Adaptive Neuro-Fuzzy model used in this study. In the first order Takagi-Sugeno fuzzy inference model, the typical fuzzy if-then rule can be expressed as:

\section{Rule 1: If $\left(v\right.$ is $\left.\mathrm{V}_{1}\right)$ And $\left(d\right.$ is $\left.\mathrm{D}_{1}\right)$ Then $f_{1}=p_{1} v+q_{1} d+t_{1}$}

Rule 2: If $\left(v\right.$ is $\left.\mathrm{V}_{2}\right)$ And $\left(d\right.$ is $\left.\mathrm{D}_{2}\right)$ Then $f_{2}=p_{2} v+q_{2} d+t_{2}$

where $V_{i}$ and $D_{i}$ are the fuzzy sets, $f_{i}$ are the outputs within the fuzzy region specified by the fuzzy rule, $p_{i}, q_{i}$ and $t_{i}$ are the design parameters that are determined during the training process. The Adaptive Neuro-Fuzzy system architecture to implement these two rules is shown in Fig. 1, in which a circle indicates a fixed node, whereas a square indicates an adaptive node.

In the first layer, all the nodes are adaptive nodes. The outputs of Layer 1 are the fuzzy membership grade of the inputs, which are given by:

$$
\begin{array}{ll}
\mathrm{O}_{1, i}=\mu_{\mathrm{Vi}}(v) & i=1,2 \\
\mathrm{O}_{1, i}=\mu_{\mathrm{Di}}(d) & i=3,4
\end{array}
$$

where $\mu_{\mathrm{V} i}(v), \mu_{\mathrm{Di}}(d)$ can adopt any fuzzy membership function. For example, if the Gaussian membership function is employed, $\mu_{\mathrm{Vi}}(v)$ is given by:

$$
\mu_{\mathrm{V}_{\mathrm{i}}}(v)=e^{\frac{-\left(v-c_{i}\right)^{2}}{2 \sigma_{i}^{2}}}
$$

where $\left\{\sigma_{i}, c_{i}\right\}$ represent the parameter set. It is significant that if the values of these parameters set changes, the Gaussian function will be changed accordingly.

In the second layer, the nodes are fixed nodes. They are labelled with $\mathrm{M}$, indicating that they perform as a simple multiplier. The outputs of this layer can be represented as:

$$
\mathrm{O}_{2, i}=w_{i}=\mu_{\mathrm{V} i}(v) \times \mu_{\mathrm{Di}}(d) \quad i=1,2
$$

which are the so-called firing strengths of the rules.

In the third layer, the nodes are also fixed nodes. They are labelled with $\mathrm{N}$, indicating that they play a normalization role to the firing strengths from the previous layer. The outputs of this layer can be represented as:

$$
\mathrm{O}_{3, \mathrm{i}}=\bar{w}_{i}=\frac{w_{i}}{w_{1}+w_{2}} \quad i=1,2
$$

which are the so-called normalized firing strengths.

In the fourth layer, the nodes are adaptive nodes. The output of each node in this layer is simply the product of the normalized firing strength and a first order polynomial (for a first order Sugeno model). Thus, the outputs of this layer are given by:

$$
\mathrm{O}_{4, \mathrm{i}}=\bar{w}_{i} f_{i}=\bar{w}_{i}\left(p_{i} v+q_{i} d+t_{i}\right) \quad i=1,2
$$

In the fifth layer, there is only one single fixed node labelled with $\Sigma$. This node performs the summation of all incoming signals. Hence, the overall output of the model is given by:

$$
\mathrm{O}_{5, \mathrm{i}}=\sum_{i=1}^{2} \bar{w}_{i} f_{i}
$$


It can be observed that there are two adaptive layers in this architecture, namely the first layer and the fourth layer. In the first layer, there are two modifiable parameters $\left\{\sigma_{i}, c_{i}\right\}$, which are related to the input membership functions. These parameters are the so-called premise parameters. In the fourth layer, there are also three modifiable parameters $\left\{p_{i}, q_{i}, t_{i}\right\}$, pertaining to the first order polynomial. These parameters are so-called consequent parameters.

Layer 1 Layer 2 Layer 3 Layer $4 \quad$ Layer 5

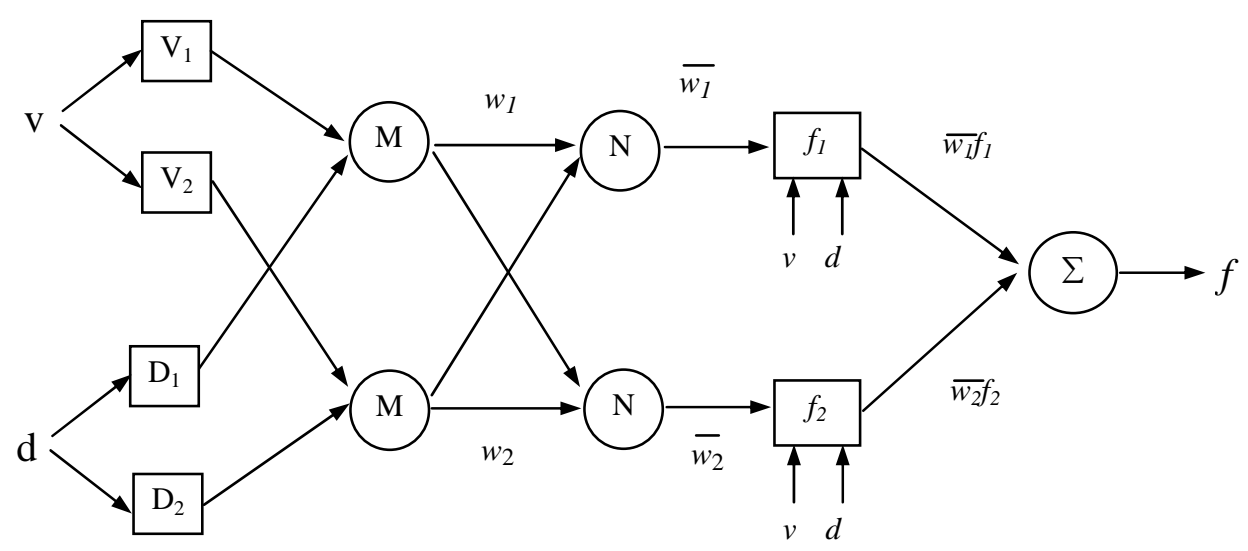

Figure 1: Basic neuro-fuzzy system architecture.

The task of the learning algorithm for this architecture is to tune all the modifiable parameters, namely $\left\{\sigma_{i}, c_{i}\right\}$ and $\left\{p_{i}, q_{i}, t_{i}\right\}$, to make the adaptive neuro-fuzzy system output match the training data. When the premise parameters $\sigma_{i}$ and $c_{i}$ of the membership function are fixed, the output of the adaptive neuro-fuzzy model can be written as:

$$
f=\bar{w}_{1} f_{1}+\bar{w}_{2} f_{2}
$$

Substituting the fuzzy if-then rules into Eq. (8), it becomes:

$$
f=\bar{w}_{1}\left(p_{1} v+q_{1} d+t_{1}\right)+\bar{w}_{2}\left(p_{2} v+q_{2} d+t_{2}\right)
$$

After rearrangement, the output can be expressed as:

$$
f=\left(\bar{w}_{1} v\right) p_{1}+\left(\bar{w}_{1} d\right) q_{1}+\left(\bar{w}_{1}\right) t_{1}+\left(\bar{w}_{2} v\right) p_{2}+\left(\bar{w}_{2} d\right) q_{2}+\left(\bar{w}_{2}\right) t_{2}
$$

which is a linear combination of the modifiable consequent parameters $p_{1}, q_{1}, t_{1}, p_{2}, q_{2}$ and $t_{2}$. The least squares method can be used to identify the optimal values of these parameters easily. When the premise parameters are not fixed, the search space becomes larger and the convergence of the training becomes slower. A hybrid algorithm combining the least squares method and the gradient descent method is adopted to solve this problem. The hybrid algorithm is composed of a forward pass and a backward pass. The least squares method (forward pass) is used to optimize the consequent parameters with the premise parameters fixed. Once the optimal consequent parameters are found, the backward pass starts immediately. The gradient descent method (backward pass) is used to adjust optimally the premise parameters corresponding to the fuzzy sets in the input domain. The output of the 
adaptive neuro-fuzzy system is calculated by employing the consequent parameters found in the forward pass. The output error is used to adapt the premise parameters by means of a standard back-propagation algorithm. In the present study the proposed neuro-fuzzy model was trained with the back-propagation gradient descent method in combination with the least squares method when cutting speed, feed rate and drill diameter were used as inputs.

\section{RESULTS AND DISCUSSION}

A training database with regard to process parameters and drill performance are required to train the fuzzy system for the modelling of drilling process. A number of drilling experiments were carried out by Lee [6] on a CNC machining centre (First MCV-641) using HSS twist drills for the machining of S45C steel plates. The drilling process parameters were selected by varying the drill diameter in the range of $8-12 \mathrm{~mm}$, the cutting speed in the range of 10-30 $\mathrm{m} / \mathrm{min}$, and feed rate in the range of $0.06-0.24 \mathrm{~mm} / \mathrm{rev}$. In the experiments, 25 drilling operation were performed based on above cutting parameter combinations. The drill life is defined as the period of drilling time until the average flank wear $V_{B}$ is equal to $0.3 \mathrm{~mm}$ or the maximum flank wear land $V_{B \text { max }}$ is equal to $0.6 \mathrm{~mm}$. In the experiments, the flank wear land was measured on both cutting edges of the drill using a tool microscope. The mean flank wear land $V_{B}$ was calculated by averaging six places of the flank wear land on the cutting edges. The thrust force and torque were measured using a dynamometer (Kistler 9271A) under the workpiece. When the drill fully entered the workpiece, the steady-state portions of the thrust force and torque signal were obtained.

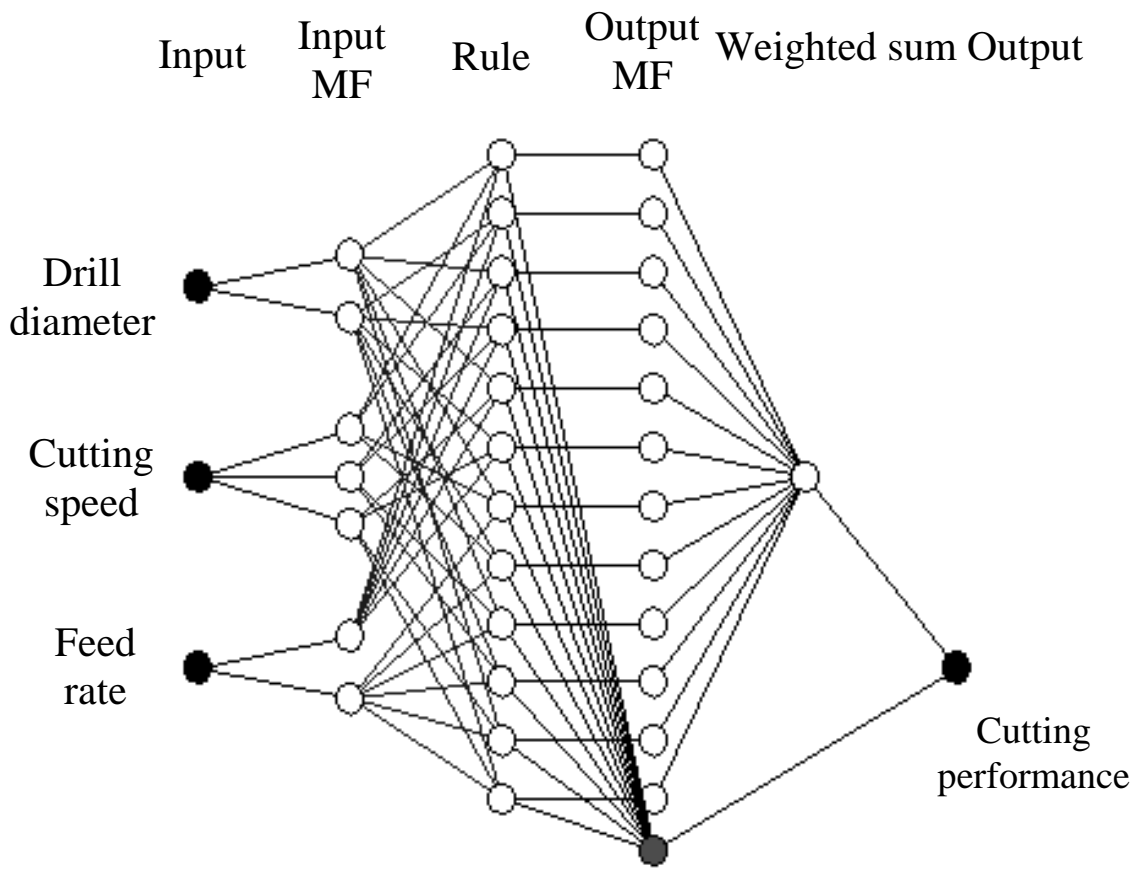

Figure 2: Schematic of neuro-fuzzy system structure.

The tool life, torque and thrust force corresponding to 25 drilling experiments is listed in Table I, which is used as the training data in proposed system for modelling. The schematic of the structure of the neuro-fuzzy system used in this study is shown in Fig. 2. Fig. 3 shows the flowchart for modelling the cutting performance using neuro-fuzzy system. 


\subsection{Modelling of tool life}

In this study, drill diameter, cutting speed, and feed rate are inputs and tool life is the output of the system. First order Sugeno fuzzy inference system with Gaussian membership function distribution for the input variables is used. The fuzzy expressions for drill diameter are: SSDSmall Size Drill, and LSD-Large Size Drill. Fuzzy expressions for cutting speed are: SS-Slow Speed, MS-Medium Speed, and HS-High Speed. Fuzzy expressions for feed rate are: LFLow Feed, and HF-High Feed.

Table I: Experimental data for training.

\begin{tabular}{|c|c|c|c|c|c|}
\hline $\begin{array}{c}\text { Drill } \\
\text { diameter } \\
(\mathrm{mm})\end{array}$ & $\begin{array}{c}\text { Cutting } \\
\text { speed } \\
(\mathrm{m} / \mathrm{min})\end{array}$ & $\begin{array}{c}\text { Feed } \\
\text { rate } \\
(\mathrm{mm} / \mathrm{rev})\end{array}$ & $\begin{array}{c}\text { Tool } \\
\text { life } \\
(\mathrm{sec})\end{array}$ & $\begin{array}{c}\text { Torque } \\
(\mathrm{Ncm})\end{array}$ & $\begin{array}{c}\text { Thrust } \\
\text { force } \\
(\mathrm{N})\end{array}$ \\
\hline 8 & 10 & 0.06 & 528 & 223 & 98 \\
\hline 8 & 10 & 0.15 & 30 & 407 & 128 \\
\hline 8 & 20 & 0.06 & 226 & 175 & 87 \\
\hline 8 & 20 & 0.15 & 45 & 359 & 153 \\
\hline 8 & 20 & 0.24 & 38 & 576 & 225 \\
\hline 8 & 30 & 0.06 & 176 & 178 & 88 \\
\hline 8 & 30 & 0.15 & 40 & 378 & 145 \\
\hline 8 & 30 & 0.24 & 31 & 546 & 216 \\
\hline 10 & 10 & 0.06 & 940 & 298 & 116 \\
\hline 10 & 10 & 0.15 & 527 & 663 & 206 \\
\hline 10 & 10 & 0.24 & 60 & 957 & 279 \\
\hline 10 & 20 & 0.06 & 282 & 288 & 117 \\
\hline 10 & 20 & 0.15 & 75 & 619 & 203 \\
\hline 10 & 20 & 0.24 & 59 & 870 & 266 \\
\hline 10 & 30 & 0.06 & 188 & 271 & 124 \\
\hline 10 & 30 & 0.15 & 63 & 538 & 181 \\
\hline 10 & 30 & 0.24 & 39 & 813 & 272 \\
\hline 12 & 10 & 0.06 & 340 & 424 & 175 \\
\hline 12 & 10 & 0.15 & 136 & 842 & 237 \\
\hline 12 & 20 & 0.06 & 283 & 400 & 152 \\
\hline 12 & 20 & 0.15 & 68 & 803 & 229 \\
\hline 12 & 20 & 0.24 & 43 & 1139 & 334 \\
\hline 12 & 30 & 0.06 & 170 & 371 & 148 \\
\hline 12 & 30 & 0.15 & 60 & 746 & 234 \\
\hline 12 & 30 & 0.24 & 38 & 1087 & 313 \\
\hline & & & & & \\
\hline
\end{tabular}

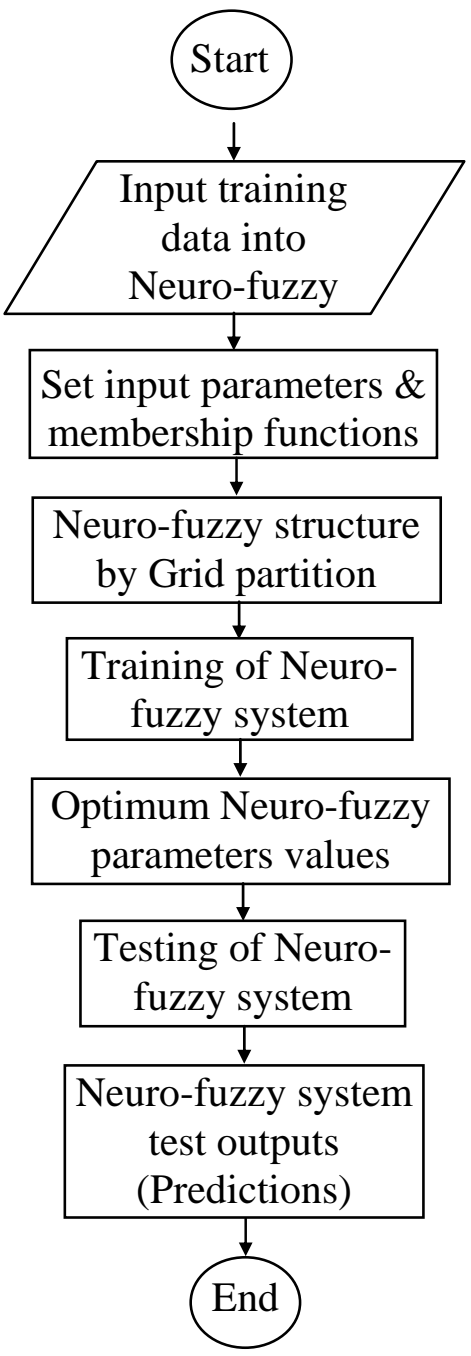

Figure 3: Flowchart of neuro-fuzzy system.

The number of fuzzy rules in a fuzzy system is related to the number of fuzzy sets for each input variables. As there are three inputs of the fuzzy system, namely drill diameter, cutting speed, and feed rate, which are classified into 2, 3 and 2 fuzzy sets respectively. 
Roy: Modelling of Tool Life, Torque and Thrust Force in Drilling: a Neuro-Fuzzy Approach

Table II: Premise parameters of fuzzy system for tool life.

\begin{tabular}{|c|c|c|}
\hline \multicolumn{3}{|l|}{ Drill diameter } \\
\hline & $\sigma_{i}$ & $c_{i}$ \\
\hline SSD & 1.6986 & 8.01 \\
\hline LSD & 1.6986 & 12.0 \\
\hline Cutting speed \\
\hline SS & 4.2466 & 10.0 \\
\hline MS & 4.2465 & 20.0 \\
\hline HS & 4.2476 & 30.0 \\
\hline Feed rate \\
\hline LF & 0.0764 & 0.06 \\
\hline HF & 0.0763 & 0.24 \\
\hline
\end{tabular}

Table III: Consequent parameters of fuzzy system for tool life.

\begin{tabular}{|c|c|c|c|c|}
\hline $\begin{array}{c}\text { Rule } \\
\text { no. }\end{array}$ & $p_{i}$ & $q_{i}$ & $r_{i}$ & $t_{i}$ \\
\hline 1 & 391.954 & -255.100 & 442.986 & -25.626 \\
\hline 2 & 257.654 & -265.102 & -450.911 & -26.566 \\
\hline 3 & 4.939 & 21.277 & -1225.285 & 0.257 \\
\hline 4 & -8.703 & 1.078 & 1225.103 & -0.770 \\
\hline 5 & 12.870 & 3.684 & -775.667 & 0.161 \\
\hline 6 & 2.161 & -6.315 & 775.626 & -0.191 \\
\hline 7 & -227.721 & 294.987 & 440.235 & 29.544 \\
\hline 8 & -192.771 & 219.318 & -433.263 & 22.038 \\
\hline 9 & 11.041 & 4.536 & -1371.92 & 1.140 \\
\hline 10 & -1.064 & -20.014 & 1371.901 & -0.310 \\
\hline 11 & -7.711 & 10.015 & -470.998 & 0.318 \\
\hline 12 & -4.189 & -0.275 & 471.002 & -0.044 \\
\hline
\end{tabular}

Table IV: Comparison of predicted tool life and experimental value.

\begin{tabular}{|c|c|c|c|c|c|}
\hline \multirow{2}{*}{$\begin{array}{c}\text { Drill } \\
\text { diameter } \\
(\mathrm{mm})\end{array}$} & \multirow{2}{*}{$\begin{array}{l}\text { Cutting } \\
\text { speed } \\
\text { (m/min) }\end{array}$} & \multirow{2}{*}{$\begin{array}{l}\text { Feed rate } \\
(\mathrm{mm} / \mathrm{rev})\end{array}$} & \multirow{2}{*}{$\begin{array}{c}\text { Tool life (sec) } \\
\text { Experimental value }\end{array}$} & \multicolumn{2}{|c|}{ Neuro-Fuzzy System } \\
\hline & & & & $\begin{array}{l}\text { Predicted Tool life } \\
\text { (sec) }\end{array}$ & Abs \% error \\
\hline 8 & 10 & 0.06 & 528 & 528.1 & 0.019 \\
\hline 8 & 10 & 0.15 & 30 & 30.2 & 0.667 \\
\hline 8 & 20 & 0.06 & 226 & 226.58 & 0.257 \\
\hline 8 & 20 & 0.15 & 45 & 44.02 & 2.178 \\
\hline 8 & 20 & 0.24 & 38 & 38.49 & 1.289 \\
\hline 8 & 30 & 0.06 & 176 & 175.63 & 0.210 \\
\hline 8 & 30 & 0.15 & 40 & 40.74 & 1.850 \\
\hline 8 & 30 & 0.24 & 31 & 30.64 & 1.161 \\
\hline 10 & 10 & 0.06 & 940 & 940.3 & 0.032 \\
\hline 10 & 10 & 0.15 & 527 & 526.6 & 0.076 \\
\hline 10 & 10 & 0.24 & 60 & 60.13 & 0.217 \\
\hline 10 & 20 & 0.06 & 282 & 280.92 & 0.383 \\
\hline 10 & 20 & 0.15 & 75 & 77.02 & 2.693 \\
\hline 10 & 20 & 0.24 & 59 & 57.97 & 1.746 \\
\hline 10 & 30 & 0.06 & 188 & 188.72 & 0.383 \\
\hline 10 & 30 & 0.15 & 63 & 61.56 & 2.286 \\
\hline 10 & 30 & 0.24 & 39 & 39.72 & 1.846 \\
\hline 12 & 10 & 0.06 & 340 & 338.47 & 0.450 \\
\hline 12 & 10 & 0.15 & 136 & 136.1 & 0.073 \\
\hline 12 & 20 & 0.06 & 283 & 283.49 & 0.173 \\
\hline 12 & 20 & 0.15 & 68 & 67.02 & 1.441 \\
\hline 12 & 20 & 0.24 & 43 & 43.49 & 1.141 \\
\hline 12 & 30 & 0.06 & 170 & 169.61 & 0.229 \\
\hline 12 & 30 & 0.15 & 60 & 61.52 & 2.534 \\
\hline 12 & 30 & 0.24 & 38 & 36.63 & 3.605 \\
\hline
\end{tabular}


Therefore, maximum number of rules for this system can be 12 . Thus, a typical $i^{\text {th }}$ rule of the fuzzy system will look as follows:

IF drill diameter $\left(d_{i}\right)$ is Small Size Drill (SSD) AND cutting speed $\left(v_{i}\right)$ is High Speed (HS) AND feed rate $\left(f_{i}\right)$ is Medium Feed (MF) THEN tool life is $\left(p_{i} v_{i}+q_{i} f_{i}+r_{i} d_{i}+t_{i}\right)$, where $p_{i}, q_{i}, r_{i}$, $t_{i}$ are the design parameters referred as consequent parameters.

During training in neuro-fuzzy inference systems, 25 sets of experimental data were used to conduct 450 cycles of learning. Neural network based hybrid-learning scenario for modelling tool life is as follows:

Number of nodes $=44$, Number of linear parameters $=48$, Number of non-linear parameters $=14$, Total number of parameters $=62$.

The values of parameters of the membership function for the fuzzy set i.e. premise parameters obtained after training for the modelling tool life are given in Table II. Table III shows the consequent parameters obtained after training. In Table IV, comparisons are made of the results of the proposed neuro-fuzzy system with those of real experiments [6] for making modelling of tool life in drilling. Simulation results indicate that proposed neuralfuzzy modelling yields on average error of $1.10 \%$ i.e. the accuracy is $98.90 \%$.

\subsection{Modelling of torque}

In this study, output of the system is torque whereas drill diameter, cutting speed, and feed rate are inputs. A typical $i^{\text {th }}$ rule of the fuzzy system will look as follows:

IF drill diameter $\left(d_{i}\right)$ is Small Size Drill (SSD) AND cutting speed $\left(v_{i}\right)$ is High Speed (HS) AND feed rate $\left(f_{i}\right)$ is Medium Feed (MF) THEN torque is $\left(p_{t i} v_{i}+q_{t i} f_{i}+r_{t i} d_{i}+t_{t i}\right)$, where $p_{t i}, q_{t i}$, $r_{t i}, t_{t i}$ are the consequent parameters.

Table V: Premise parameters of fuzzy system for torque.

\begin{tabular}{|c|c|c|}
\hline \multicolumn{3}{|c|}{ Drill diameter } \\
\hline & $\sigma_{i}$ & $C_{i}$ \\
\hline SSD & 1.6781 & 7.9786 \\
\hline LSD & 1.7162 & 11.9801 \\
\hline Cutting speed \\
\hline SS & 4.2467 & 10.00 \\
\hline MS & 4.2464 & 20.00 \\
\hline HS & 4.2466 & 29.99 \\
\hline \multicolumn{3}{|c|}{ Feed rate } \\
\hline LF & 0.0215 & 0.0296 \\
\hline HF & 0.0841 & 0.2272 \\
\hline
\end{tabular}

Table VI: Consequent parameters of fuzzy system for torque.

\begin{tabular}{|c|c|c|c|c|}
\hline $\begin{array}{c}\text { Rule } \\
\text { no. }\end{array}$ & $p_{t i}$ & $q_{t i}$ & $r_{t i}$ & $t_{t i}$ \\
\hline 1 & -18.586 & 41.641 & 0.248 & 4.152 \\
\hline 2 & 32.023 & -35.184 & 3142.079 & -3.507 \\
\hline 3 & 1.312 & 6.637 & 0.027 & 0.457 \\
\hline 4 & 16.235 & -5.332 & 2189.068 & -0.382 \\
\hline 5 & 2.766 & 4.361 & 0.008 & 0.149 \\
\hline 6 & -10.938 & 5.891 & 1770.291 & 0.192 \\
\hline 7 & 41.355 & -9.338 & -0.059 & -0.976 \\
\hline 8 & -7.610 & 45.013 & 3416.245 & 4.456 \\
\hline 9 & 10.123 & 13.560 & 0.037 & 0.635 \\
\hline 10 & -7.984 & 17.149 & 3711.862 & 0.996 \\
\hline 11 & 2.934 & 10.915 & 0.022 & 0.378 \\
\hline 12 & 13.217 & -0.110 & 3991.652 & 0.0114 \\
\hline
\end{tabular}

Neural network based hybrid-learning scenario for modelling torque is as follows:

Number of nodes $=44$, Number of linear parameters $=48$, Number of non-linear parameters $=14$, Total number of parameters $=62$. 
The values of parameters of the membership function for the fuzzy sets i.e. premise parameters obtained after training for the modelling thrust force are given in Table V. Table VI shows the consequent parameters obtained after training. In Table VII, comparisons are made of the results of the proposed neural-fuzzy system with those of real experiments [6] for making modelling of torque in drilling. Results indicate that proposed neuro-fuzzy modelling yields on average error of $0.42 \%$.

Table VII: Comparison of predicted torque and experimental value.

\begin{tabular}{|c|c|c|c|c|c|}
\hline \multirow{2}{*}{$\begin{array}{c}\text { Drill diameter } \\
(\mathrm{mm})\end{array}$} & \multirow{2}{*}{$\begin{array}{l}\text { Cutting } \\
\text { speed } \\
\text { (m/min) }\end{array}$} & \multirow{2}{*}{$\begin{array}{l}\text { Feed rate } \\
(\mathrm{mm} / \mathrm{rev})\end{array}$} & \multirow{2}{*}{$\begin{array}{l}\text { Torque }(\mathrm{Ncm}) \\
\text { Experimental } \\
\text { value }\end{array}$} & \multicolumn{2}{|c|}{ Neuro-Fuzzy System } \\
\hline & & & & $\begin{array}{l}\text { Predicted Torque } \\
\qquad(\mathrm{Ncm})\end{array}$ & Abs \% error \\
\hline 8 & 10 & 0.06 & 223 & 222.49 & 0.2287 \\
\hline 8 & 10 & 0.15 & 407 & 407.131 & 0.0322 \\
\hline 8 & 20 & 0.06 & 175 & 174.41 & 0.337 \\
\hline 8 & 20 & 0.15 & 359 & 363.384 & 1.221 \\
\hline 8 & 20 & 0.24 & 576 & 571.616 & 0.761 \\
\hline 8 & 30 & 0.06 & 178 & 178.302 & 0.1696 \\
\hline 8 & 30 & 0.15 & 378 & 374.986 & 0.797 \\
\hline 8 & 30 & 0.24 & 546 & 549.014 & 0.552 \\
\hline 10 & 10 & 0.06 & 298 & 298.01 & 0.0034 \\
\hline 10 & 10 & 0.15 & 663 & 663.073 & 0.011 \\
\hline 10 & 10 & 0.24 & 957 & 956.926 & 0.0076 \\
\hline 10 & 20 & 0.06 & 288 & 287.9 & 0.0347 \\
\hline 10 & 20 & 0.15 & 619 & 610.075 & 1.442 \\
\hline 10 & 20 & 0.24 & 870 & 878.924 & 1.0257 \\
\hline 10 & 30 & 0.06 & 271 & 270.89 & 0.0406 \\
\hline 10 & 30 & 0.15 & 538 & 544.203 & 1.1531 \\
\hline 10 & 30 & 0.24 & 813 & 806.796 & 0.763 \\
\hline 12 & 10 & 0.06 & 424 & 423.79 & 0.0495 \\
\hline 12 & 10 & 0.15 & 842 & 842.13 & 0.015 \\
\hline 12 & 20 & 0.06 & 400 & 399.7 & 0.075 \\
\hline 12 & 20 & 0.15 & 803 & 807.604 & 0.573 \\
\hline 12 & 20 & 0.24 & 1139 & 1134.395 & 0.4043 \\
\hline 12 & 30 & 0.06 & 371 & 370.9 & 0.0269 \\
\hline 12 & 30 & 0.15 & 746 & 742.874 & 0.419 \\
\hline 12 & 30 & 0.24 & 1087 & 1090.125 & 0.2875 \\
\hline
\end{tabular}

\subsection{Modelling of thrust force}

In this study, drill diameter, cutting speed, and feed rate are inputs and thrust force is the output of the system. The maximum number of rules for this system is 12 . Thus, a typical $i^{\text {th }}$ rule of the fuzzy system will look as follows:

IF drill diameter $\left(d_{i}\right)$ is Small Size Drill (SSD) AND cutting speed $\left(v_{i}\right)$ is High Speed (HS) AND feed rate $\left(f_{i}\right)$ is Medium Feed (MF) THEN thrust force is $\left(P_{i} v_{i}+Q_{i} f_{i}+R_{i} d_{i}+T_{i}\right)$, where $P_{i}$, $Q_{i}, R_{i}, T_{i}$ are the consequent parameters. 
Roy: Modelling of Tool Life, Torque and Thrust Force in Drilling: a Neuro-Fuzzy Approach

Table VIII: Premise parameters of fuzzy system for thrust force.

\begin{tabular}{|c|c|c|}
\hline \multicolumn{3}{|c|}{ Drill diameter } \\
\hline & $\sigma_{i}$ & $c_{i}$ \\
\hline SSD & 1.6994 & 8.0 \\
\hline LSD & 1.6978 & 12.0 \\
\hline Cutting speed \\
\hline SS & 4.2466 & 10.0 \\
\hline MS & 4.2466 & 20.0 \\
\hline HS & 4.2466 & 30.0 \\
\hline Feed rate \\
\hline LF & 0.0845 & 0.0726 \\
\hline HF & 0.0390 & 0.2654 \\
\hline
\end{tabular}

Table IX: Consequent parameters of fuzzy system for thrust force.

\begin{tabular}{|c|c|c|c|c|}
\hline $\begin{array}{c}\text { Rule } \\
\text { no. }\end{array}$ & $P_{i}$ & $Q_{i}$ & $R_{i}$ & $T_{i}$ \\
\hline 1 & -10.877 & 15.619 & 433.500 & 1.569 \\
\hline 2 & 1536.453 & -1303.627 & -39.860 & -130.914 \\
\hline 3 & 5.767 & -1.215 & 801.573 & -0.013 \\
\hline 4 & -94.410 & 93.635 & -15.324 & 0.585 \\
\hline 5 & -0.193 & 1.955 & 524.464 & 0.062 \\
\hline 6 & 14.556 & -0.494 & -10.078 & 0.166 \\
\hline 7 & 13.941 & -7.135 & 1547.844 & -0.735 \\
\hline 8 & -1235.292 & 1061.868 & -4.216 & 106.594 \\
\hline 9 & -2.155 & 6.554 & 853.605 & 0.301 \\
\hline 10 & 101.252 & -62.217 & -16.418 & 0.207 \\
\hline 11 & 1.777 & 2.474 & 907.942 & 0.089 \\
\hline 12 & -8.324 & 16.071 & -17.420 & 0.401 \\
\hline
\end{tabular}

Table X: Comparison of predicted thrust force and experimental value.

\begin{tabular}{|c|c|c|c|c|c|}
\hline \multirow{2}{*}{$\begin{array}{l}\text { Drill diameter } \\
\qquad(\mathrm{mm})\end{array}$} & \multirow{2}{*}{$\begin{array}{l}\text { Cutting } \\
\text { speed } \\
(\mathrm{m} / \mathrm{min})\end{array}$} & \multirow{2}{*}{$\begin{array}{l}\text { Feed rate } \\
(\mathrm{mm} / \mathrm{rev})\end{array}$} & \multirow{2}{*}{$\begin{array}{c}\text { Thrust force }(\mathrm{N}) \\
\text { Experimental } \\
\text { value }\end{array}$} & \multicolumn{2}{|c|}{ Neuro-Fuzzy System } \\
\hline & & & & $\begin{array}{l}\text { Predicted Thrust } \\
\text { force }(\mathrm{N})\end{array}$ & Abs \% error \\
\hline 8 & 10 & 0.06 & 98 & 97.81 & 0.194 \\
\hline 8 & 10 & 0.15 & 128 & 128.15 & 0.117 \\
\hline 8 & 20 & 0.06 & 87 & 84.54 & 2.827 \\
\hline 8 & 20 & 0.15 & 153 & 155.51 & 1.640 \\
\hline 8 & 20 & 0.24 & 225 & 224.84 & 0.071 \\
\hline 8 & 30 & 0.06 & 88 & 90.42 & 2.750 \\
\hline 8 & 30 & 0.15 & 145 & 142.52 & 1.710 \\
\hline 8 & 30 & 0.24 & 216 & 216.35 & 0.162 \\
\hline 10 & 10 & 0.06 & 116 & 116.18 & 0.155 \\
\hline 10 & 10 & 0.15 & 206 & 205.81 & 0.092 \\
\hline 10 & 10 & 0.24 & 279 & 279.1 & 0.036 \\
\hline 10 & 20 & 0.06 & 117 & 121.92 & 4.205 \\
\hline 10 & 20 & 0.15 & 203 & 197.97 & 2.478 \\
\hline 10 & 20 & 0.24 & 266 & 266.11 & 0.041 \\
\hline 10 & 30 & 0.06 & 124 & 119.16 & 3.903 \\
\hline 10 & 30 & 0.15 & 181 & 185.94 & 2.729 \\
\hline 10 & 30 & 0.24 & 272 & 271.89 & 0.040 \\
\hline 12 & 10 & 0.06 & 175 & 174.92 & 0.046 \\
\hline 12 & 10 & 0.15 & 237 & 237.08 & 0.034 \\
\hline 12 & 20 & 0.06 & 152 & 149.55 & 1.612 \\
\hline 12 & 20 & 0.15 & 229 & 231.5 & 1.092 \\
\hline 12 & 20 & 0.24 & 334 & 333.94 & 0.018 \\
\hline 12 & 30 & 0.06 & 148 & 150.42 & 1.635 \\
\hline 12 & 30 & 0.15 & 234 & 231.52 & 1.059 \\
\hline 12 & 30 & 0.24 & 313 & 313.35 & 0.112 \\
\hline
\end{tabular}


During training in neural-fuzzy systems, 25 sets of experimental data were used to conduct 300 cycles of learning. Neural network based hybrid-learning scenario for modelling thrust force is as follows:

Number of nodes $=44$, Number of linear parameters $=48$, Number of non-linear parameters $=14$, Total number of parameters $=62$.

The values of parameters of the membership function for the fuzzy sets i.e. premise parameters obtained after training for the modelling thrust force are given in Table VIII. Table IX shows the consequent parameters obtained after training. In Table $\mathrm{X}$, comparisons are made of the results of the proposed neural-fuzzy system with those of real experiments [6] for making modelling of thrust force in drilling. Results indicate that proposed neuro-fuzzy modelling yields on average error of $1.15 \%$ i.e. the accuracy is $98.85 \%$. Therefore, more accurate results are obtained from a model constructed with neural-fuzzy system to model tool life, torque and thrust force.

\section{CONCLUSION}

The paper has described the use of neural networks and fuzzy inference system to model tool life, torque and thrust force in drilling operation. Using combination of back-propagation algorithm and least squares estimation, neural networks tuned fuzzy inference system can automatically and effectively generate the membership functions and rule base, which exempt the design of fuzzy inference system from heavy reliance on human experts. The outputs of the proposed system have been compared with the experimental results. The comparison indicates that the proposed system can produce optimal knowledge base of the fuzzy inference system for predicting tool life, torque and thrust force in drilling operation. The ability of modelling and predicting outputs of a machining process using neuro-fuzzy approach will help us to develop intelligent manufacturing system. The proposed neuro-fuzzy approach can also be used to model process performances of other conventional, unconventional machining processes and micro machining process.

\section{ACKNOWLEDGEMENTS}

The author acknowledges all support from Department of Mechanical Engineering, NIT, Durgapur. The author is grateful to Prof. D. K. Pratihar, IIT, Kharagpur, for his guidance.

\section{REFERENCES}

[1] Choudhury, S. K.; Raju, G. (2000). Investigation into crater wear in drilling, International Journal of Machine Tools and Manufacture, Vol. 40, 887-898

[2] Xiaoli, L.; Tso, S. K. (1999). Drill wear monitoring based on current signals, Wear, Vol. 231, $172-178$

[3] Ertunc, H. M.; Oysu, C. (2004). Drill wear monitoring using cutting force signal, Mechatronics, Vol. 14, 533-548

[4] Liu, H. S.; Lee, B. Y.; Tarng, Y. S. (2000). In-process prediction of corner wear in drilling operations, Journal of Materials Processing Technology, Vol. 101, 152-158

[5] Lin, S. C.; Ting, C. J. (1995). Tool wear monitoring in drilling using force signals, Wear, Vol. 180, 53-60

[6] Lee, B. Y.; Liu, H. S.; Tarng, Y. S. (1998). Modeling and optimization of drilling process, Journal of Materials Processing Technology, Vol. 74, 149-157 
[7] Lin, S. C.; Ting, C. J. (1996). Drill wear monitoring using neural network, International Journal of Machine Tools and Manufacture, Vol. 36, 465-475

[8] Panda, S. S.; Singh, A. K.; Chakraborty, D.; Pal, S. K. (2006). Drill wear monitoring using back propagation neural network, Journal of Materials Processing Technology, Vol. 172, 283-290

[9] Singh, A. K.; Panda, S. S.; Pal, S. K.; Chakraborty, D. (2006). Predicting drill wear using an artificial neural network, International Journal of Advanced Manufacturing Technology, Vol. 28, 456-462

[10] Panda, S. S.; Chakraborty, D.; Pal, S. K. (2008). Drill wear prediction using different neural network architectures, International Journal of Knowledge-based and Intelligent Engineering Systems, Vol. 12, No. 5-6, 327-338

[11] Tsao, C. C. (2002). Prediction of flank wear of different coated drills for JIS SUS 304 stainless steel using neural network, Journal of Materials Processing Technology, Vol. 123, 354-360

[12] Tsao, C. C.; Hocheng, H. (2008). Evaluation of thrust force and surface roughness in drilling composite material using Taguchi analysis and neural network, Journal of Materials Processing Technology, Vol. 203, 342-348

[13] Panda, S. S.; Chakraborty, D.; Pal, S. K. (2007). Monitoring of drill flank wear using fuzzy backpropagation neural network, International Journal of Advanced Manufacturing Technology, Vol. 34, No. 3-4, 227-235

[14] Sanjay, C.; Neema, M. L.; Chin, C. W. (2005). Modeling of tool wear in drilling by statistical analysis and artificial neural network, Journal of Materials Processing Technology, Vol. 170, 494-500

[15] Panda, S. S.; Chakraborty, D.; Pal, S. K. (2008). Flank wear prediction in drilling using back propagation neural network and radial basis function network, Applied Soft Computing, Vol. 8, 858-871

[16] Klir, G. J.; Yuan, B. (2001). Fuzzy Sets and Fuzzy Logic: Theory and Applications, Prentice-Hall of India Private Limited, India

[17] Takagi, T.; Sugeno, M. (1985). Fuzzy identification of systems and its applications to modeling and control, IEEE Transactions on Systems, Man, and Cybernetics, Vol. 15, No. 1, 116-132

[18] Jang, J. S. R. (1993). ANFIS: Adaptive-network-based fuzzy inference system, IEEE Transactions on Systems, Man, and Cybernetics, Vol. 23, No. 3, 665-685 